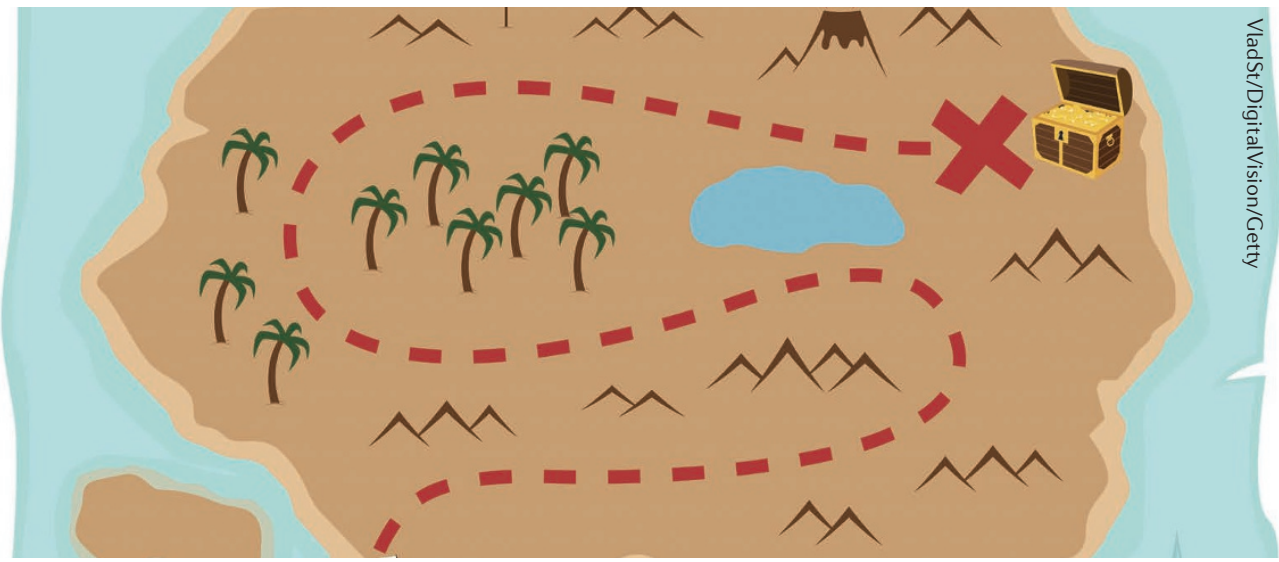

$\Rightarrow$ TRANSCRIPTION

\title{
Putting R loops firmly on the map
}

Two papers present novel techniques for more precisely mapping $\mathrm{R}$ loops - hybrid nucleic acid structures in which RNA is hybridized to one strand of DNA while the other strand of DNA is displaced and 'loops out'. Chen et al. describe a chromatin immunoprecipitation (ChIP) technique, termed R-ChIP, which uses a catalytically inactive $\mathrm{RNase} \mathrm{H}$ to immunoprecipitate R loops. Dumelie and Jaffrey report a technique called bisulfite DNA-RNA immunoprecipitation sequencing (bisDRIP-seq), in which bisulfite converts cytosines to uracils in genomic regions of single-stranded DNA before DRIP-seq.

In developing R-ChIP, Chen et al. expressed a V5-tagged catalytically inactive RNase $\mathrm{H} 1$ which has high affinity for $\mathrm{R}$ loops in vivo - in HEK293T cells and carried out ChIP with a V5-targeted antibody. The single-stranded DNA hybridized to RNA in the captured DNA-RNA hybrids was then converted to double-stranded DNA, and the resulting R-ChIP libraries were subjected to deep sequencing. Relative to the established DRIP-seq approach, in which RNADNA hybrids are directly captured by the S9.6 antibody prior to sequencing, initial analysis revealed that $\mathrm{R}$ loops identified using $\mathrm{R}$-ChIP have a stronger GC skew (a high GC ratio in the non-template DNA strand, which is typical of $R$ loops), greater linkage with transcription and increased association with open chromatin. Thus, R-ChIP seems to be more robust than DRIP-seq. Further analysis revealed that $59.3 \%$ of R loops map to promoter-proximal regions, and, using an inhibitor of transcription in combination with $\mathrm{R}$-ChIP, the authors showed that the induction of $\mathrm{R}$ loops correlates with transcriptional pausing at transcription start sites (TSSs). Finally, after observing that the majority of R-ChIP-mapped R loops are associated with a nearby free RNA end, the authors used engineered ribozymes to show that a free RNA end, coupled with an $\mathrm{R}$-loop-promoting sequence, is sufficient to generate $\mathrm{R}$ loops.

In bisDRIP-seq, Dumelie and Jaffrey lysed MCF7 cells in the presence of bisulfite, which converts cytosine to uracil in the single-stranded DNA portion of R loops, followed by DRIP-seq. A computational pipeline identified single-stranded regions with high concentrations of uracil; a prominence of cytosine-uracil conversions on one strand of DNA indicates the presence of an R loop. bisDRIP-seq scores were calculated for individual nucleotides, and the authors found that, in line with DRIP-seq scores, these scores were enriched in the regions of active promoters. Interestingly, bisDRIP-seq data suggested that TSSs, which surround R loops, are the 5 ' boundary of promoter-associated $R$ loops and that the first exon-intron junction of a gene acts as the 3' boundary of R loops. As promoter regions of intronless genes were strongly associated with $\mathrm{R}$ loops, the exon-intron junction must not promote, or be required for, $\mathrm{R}$-loop formation. $\mathrm{R}$ loops in intronless histone genes were all bordered at the 5 ' end by the TSS; however, as some $\mathrm{R}$ loops were restricted to the initial portion of the gene and others covered nearly the whole gene, 3' R-loop boundaries in intronless genes are variable across genes.

These new techniques for detecting $\mathrm{R}$ loops will enable researchers to study the formation of these structures, and their roles in transcription, chromatin structure and genomic instability, in more detail.

Katharine H. Wrighton

ORIGINAL ARTICLES Chen, L. et al. R-ChIP using inactive RNase H reveals dynamic coupling of $R$-loops with transcriptional pausing at gene promoters. Mol. Cell http://dx.doi.org/10.1016/

j.molcel.2017.10.008 (2017) | Dumelie, J. G. \& Jaffrey, S. R. Defining the location of promoter-associated R-loops at near-nucleotide resolution using bisDRIP-seq. eLife 6, e28306 (2017) 